\title{
DUALITY AND POLYNOMIAL TESTING OF TREE HOMOMORPHISMS
}

\author{
P. HELL, J. NEŠETřIL, AND X. ZHU
}

\begin{abstract}
Let $H$ be a fixed digraph. We consider the $H$-colouring problem, i.e., the problem of deciding which digraphs $G$ admit a homomorphism to $H$. We are interested in a characterization in terms of the absence in $G$ of certain tree-like obstructions. Specifically, we say that $H$ has tree duality if, for all digraphs $G, G$ is not homomorphic to $H$ if and only if there is an oriented tree which is homomorphic to $G$ but not to $H$. We prove that if $H$ has tree duality then the $H$-colouring problem is polynomial. We also generalize tree duality to bounded treewidth duality and prove a similar result. We relate these duality concepts to the notion of the $\underline{X}$-property studied by Gutjahr, Welzl, and Woeginger.

We then focus on the case when $H$ itself is an oriented tree. In fact, we are particularly interested in those trees that have exactly one vertex of degree three and all other vertices of degree one or two. Such trees are called triads. We have shown in a companion paper that there exist oriented triads $H$ for which the $H$-colouring problem is $N P$-complete. We contrast these with several families of oriented triads $H$ which have tree duality, or bounded treewidth duality, and hence polynomial $H$-colouring problems. If $P \neq N P$, then no oriented triad $H$ with an $N P$-complete $H$-colouring problem can have bounded treewidth duality; however no proof of this is known, for any oriented triad $H$. We prove that none of the oriented triads $H$ with $N P$-complete $H$ colouring problems given in the companion paper has tree duality.
\end{abstract}

\section{INTRODUCTION}

A homomorphism of a digraph $G$ to a digraph $H$ is a mapping of the vertex sets $f: V(G) \rightarrow V(H)$ which preserves the edges, i.e., such that $x y \in E(G)$ implies $f(x) f(y) \in E(H)$. If a homomorphism of $G$ to $H$ exists, we say $G$ is homomorphic to $H$ and write $G \rightarrow H$. Otherwise we write $G \nrightarrow H$. A digraph $G$ is a core if it is not homomorphic to any of its proper subgraphs.

Let $H$ be a fixed digraph. The $H$-colouring problem is the decision problem in which we are given an arbitrary digraph $G$ and are to decide whether or not $G$ is homomorphic to $H$. The name is due to the fact that for undirected graphs the $K_{n}$-colouring problem simply asks whether or not $G$ is $n$-colourable. It was shown in [17] that for undirected graphs $H$-colouring is polynomial when $H$ is bipartite and $N P$-complete otherwise. The $H$-colouring problem for digraphs has received much recent attention, $[3,5,6,10,11,14,15,20,21,22,23,27,28,35]$. Unlike the situation for undirected graphs, the boundary between easy and hard $H$-colouring problems for digraphs $H$ is not understood, and it is not even known

Received by the editors July 20, 1993.

1991 Mathematics Subject Classification. Primary 05C85; Secondary 68Q25. 
whether all $H$-colouring problems are polynomial or $N P$-complete. Many examples of digraphs $H$ with polynomial and with $N P$-complete $H$-colouring problems are given in $[3,4,5,6,10,11,26,27,28]$. In a companion paper [20], we exhibit oriented trees $H$ with $N P$-complete $H$-colouring problems. Welzl et al. [15] were the first to discover such an oriented tree $H$. Their tree has 287 vertices and maximum degree 5. The simple trees given in [20] are oriented triads, i.e., the underlying undirected trees have one vertex of degree three and all other vertices of degree one or two. The smallest oriented tree in this family has only 45 vertices.

Thus $H$-colouring problems can be hard even when $H$ is an oriented tree. However, there are also many oriented trees $H$ for which there is structure to the $H$-colouring problem, which can be exploited to find a polynomial algorithm. The class of digraphs homomorphic to such a "nice" oriented tree $H$ can be characterized by the absence of certain simple obstructions. A typical example is the case of oriented paths. According to [23], a digraph $G$ is homomorphic to an oriented path $H$ if and only if each oriented path $P$ homomorphic to $G$ is also homomorphic to $H$. Thus in this case the obstructions are oriented paths $P$ homomorphic to $G$ but not to $H$. To make this obstruction point of view more explicit we restate the characterization (for the case when $H$ is an oriented path) as follows: $A$ digraph $G$ is not homomorphic to $H$ if and only if there exists an oriented path $P$ which is homomorphic to $G$ but not to $H$. We shall state all our results in this "negative" form to emphasize the obstruction point of view. Another class of digraphs with a similar characterization theorem is the class of unbalanced cycles. An unbalanced cycle is an oriented cycle in which the number of forward edges is different from the number of backward edges (with respect to some fixed traversal of the cycle). According to [22], a digraph $G$ is not homomorphic to an unbalanced cycle $H$ if and only if there is an oriented cycle $C$ homomorphic to $G$ but not homomorphic to $H$. (The result in [22] gives a more specific condition from which this characterization easily follows.)

In this paper we are interested in digraphs (in particular oriented trees) $H$ for which there is a similar characterization of the class of digraphs $G$ homomorphic to $H$. Specifically, we say that $H$ has tree duality if the following property holds for all digraphs $G$ : A digraph $G$ is not homomorphic to $H$ if and only if there exists an oriented tree homomorphic to $G$ but not to $H$. We have found tree duality to be a surprisingly useful property. In particular, we shall prove that if $H$ has tree duality then the $H$-colouring problem is polynomial.

Observe that oriented paths $H$ have tree duality, by the above result from [23], and hence the $H$-colouring problem is polynomial for each oriented path $H$; this was first proved in [15].

The class of digraphs $H$ with polynomial $H$-colouring problems can be further enlarged by generalizing tree duality to treewidth- $k$ duality: An undirected graph is a $k$-tree if it can be obtained from a $k$-clique by repeatedly adding a vertex joined to $k$ existing vertices which form a $k$-clique. (Thus a tree is a 1-tree.) An undirected graph is said to have treewidth $k$, or to be a partial $k$-tree, if it is a spanning subgraph of a $k$-tree. Partial $k$-trees have been found to admit efficient algorithms for many hard computational problems [33]. We say that an oriented graph has treewidth $k$ (or is an oriented partial $k$-tree) if its uderlying undirected graph has treewidth $k$. We say a digraph $H$ has treewidth- $k$ duality if the following property holds for all digraphs $G$ : A digraph $G$ is not homomorphic to $H$ if and only if there exists an oriented partial $k$-tree homomorphic to $G$ but not to $H$. It 
is easy to see that treewidth-1 duality is equivalent to tree duality. We say that $H$ has bounded treewidth duality if it has treewidth- $k$ duality for some positive integer $k$. We shall also show that if $H$ has bounded treewidth duality then $H$-colouring is polynomial. Since cycles have treewidth 2 , we conclude from the above result from [22] that unbalanced cycles $H$ have treewidth- 2 duality, and hence the $H$-colouring problem is polynomial. This was first proved in [14] and independently [35], cf. also [10].

In general, we use the word "duality" to mean a statement which relates the non-existence of a certain homomorphism to the existence of certain other homomorphisms. To be precise, let $\mathcal{C}$ be a fixed class of digraphs. For a digraph $H$ we denote by $(\rightarrow H)_{\mathcal{C}}$ the class of digraphs from $\mathcal{C}$ which are homomorphic to $H$. For a class $\mathcal{G}$ of digraphs, we denote by $(\mathcal{G} \nrightarrow)_{\mathcal{C}}$ the class of all digraphs $H \in \mathcal{C}$ such that no member of $\mathcal{G}$ is homomorphic to $H$. When $\mathcal{G}$ consists of a single graph $G$ we write $(G \nrightarrow)_{\mathcal{C}}$ for $(\mathcal{G} \nrightarrow)_{\mathcal{C}}$, and when $\mathcal{C}$ is the class of all graphs, or digraphs, we omit the subscript $\mathcal{C}$. A duality result is a statement of the form

$$
(\rightarrow H)_{\mathcal{C}}=(\mathcal{G} \nrightarrow)_{\mathcal{C}}
$$

Many known results which are considered "duality theorems" can be presented in this format. For instance, the well known fact that an undirected graph is bipartite if and only if it contains no odd cycles, can be written as $\left(\rightarrow K_{2}\right)=$ $\left(\mathcal{C}_{\text {odd }} \nrightarrow\right)$ (where $\mathcal{C}_{\text {odd }}$ is the set of all odd cycles). The duality statement $\left(\rightarrow K_{n}\right)_{\mathcal{C}}$ $=\left(K_{n+1} \nrightarrow\right)_{\mathcal{C}}$ says that the size of the maximum clique in $G$ is equal to the chromatic number of $G$, for every graph $G \in \mathcal{C}$. Thus when $\mathcal{C}$ consists of all complements of comparability graphs we obtain Dilworth's theorem, when $\mathcal{C}$ consists of all complements of bipartite graphs we obtain Konig's theorem, and so on $([13,7])$. Nešetřil, Pultr, and Komárek [31, 25] studied duality schemes (*) in which $\mathcal{C}$ is the class of all graphs or digraphs and $\mathcal{G}$ consists of a single graph or digraph $G$. Häggkvist and Hell [16] studied duality schemes $(*)$ for the class $\mathcal{C}$ of graphs and digraphs of bounded degree. Our definition of tree duality (treewidth- $k$ duality) is easily seen to be equivalent to the statement $(\rightarrow H)=\left(\mathcal{T}_{H} \nrightarrow\right)$, where $\mathcal{T}_{H}$ is the class of all oriented trees (respectively oriented partial $k$-trees) which are not homomorphic to $H$.

Gutjahr, Welzl and Woeginger [15] introduced the concept of extended $\underline{X}$ property of a digraph. Digraphs $H$ with this property have polynomial $H$-colouring problems. Many known digraphs $H$ with polynomial $H$-colouring problems in fact do have the extended $\underline{X}$-property. However, there are also many known digraphs $H$ with polynomial $H$-colouring problems which do not have the extended $X$-property. We shall show that the class of digraphs $H$ with bounded treewidth duality (respectively of balanced digraphs with tree duality) is strictly larger than the class of digraphs (respectively balanced digraphs) with the extended $\underline{X}$-property. Moreover, we do not know of any digraph $H$ with polynomial $H$-colouring problem, which does not have bounded treewidth duality.

In the second half of the paper we focus on oriented trees. We shall prove that many classes of oriented trees, in particular of oriented triads, have tree duality. We shall also describe a family of oriented triads which have treewidth-2 duality and some members of which do not have tree duality. Therefore, all these oriented trees $H$ admit polynomial $H$-colouring algorithms. On the other hand, if $P \neq N P$ then no oriented tree $H$ with $N P$-complete $H$-colouring problem can have bounded treewidth duality. We do not have a proof of this for any oriented tree $H$. (For 
certain oriented cycles $H$, this has been proved in [32].) However, we were able to verify that many of the oriented trees $H$ with known $N P$-complete $H$-colouring problems do not have tree duality.

An oriented path $P$ in a digraph $G$ is an ordered sequence of vertices, $P=$ $\left[p_{0}, p_{1}, \ldots, p_{n}\right]$, such that for each $i=0,1, \ldots, n-1$ either $p_{i} p_{i+1}$ or $p_{i+1} p_{i}$ is an edge of $G$; the former are called forward edges of $P$, and the latter backward edges of $P$. We denote by $P^{-1}$ the oriented path $\left[p_{n}, p_{n-1}, \ldots, p_{0}\right]$; note that $P^{-1}$ is the same digraph as $P$, only the direction of its traversal has changed (and so forward edges have become backward edges and vice versa). The algebraic length al $(P)$ of an oriented path $P$ is the number of forward edges minus the number of backward edges of $P$; the net length $n l(P)$ of $P$ is the absolute value of $a l(P)$. An oriented path of net length $n$ is minimal if it contains no proper subpath of net length $n$. Oriented cycles in $G$, and their algebraic length and net length are defined analogously. A balanced cycle is an oriented cycle of net length zero. A digraph $G$ is balanced if each oriented cycle in $G$ is balanced. If $G$ is balanced then for any two vertices $x, y$ of $G$, all $x, y$-paths in $G$ have the same algebraic length; we denote this length by $d_{G}(x, y)$. Two vertices $x, y$ of a balanced digraph $G$ are said to be on the same level if $d_{G}(x, y)=0$. The height $h t(G)$ of a balanced digraph $G$ is the maximum net length of an oriented path in $G$.

\section{ThE ROLE OF DUALITY IN POLYNOMIAL HOMOMORPHISM ALGORITHMS}

In this section we prove that if a digraph $H$ has tree duality, or bounded treewidth duality, then the $H$-colouring problem is polynomial. Our proof builds on techniques developed in [15], and is related to early work on constraint satisfaction algorithms [29], and monadic second-order logic [1, 8]. To simplify the exposition we will concentrate on tree duality and only briefly mention the extensions to bounded treewidth duality.

We first describe a procedure called the consistency check. Suppose that $G$ and $H$ are digraphs, and $\left(e_{1}, e_{2}, \cdots, e_{m}\right)$ is an enumeration of the edges of $G$. Thus $m=|E(G)|$; also let $n=|V(G)|$ and $k=|V(H)|$. A labeling of $G$ with respect to $H$ is a mapping $l$ of $V(G)$ to the family of subsets of $V(H)$. (Thus a label $l(v)$ of a vertex $v \in V(G)$ is a set of vertices of $H$; we think of $l(v)$ as the set of "possible images" of the vertex $v$.) We say that a labeling $l$ is consistent with an edge $e_{i}=x y$, if for any $r \in l(x)$ there is a $s \in l(y)$ such that $r s \in E(H)$, and for any $s \in l(y)$ there is an $r \in l(x)$ such that $r s \in E(H)$. The size of a labeling $l$ of $G$ is the sum of $|l(v)|$ over all vertices of $G$. The consistency check for $G$ with respect to $H$ is the following computation of a labeling $l^{*}$ consistent with all edges:

In the 0 -th step, we let $l(v)=V(H)$ for all $v \in V(G)$. In the $j$-th step, $j=$ $1,2, \ldots, m n k$, we check if the present labeling $l$ is consistent with the edge $e_{i}=x y$ for $i \equiv j(\bmod m)$. If not, we then modify the labeling $l$ by removing from $l(y)$ those labels $s$ for which there is no $r \in l(x)$ with $r s \in E(H)$, and removing from $l(x)$ those labels $r$ for which there is no $s \in l(y)$ with $r s \in E(H)$. The resulting labeling is then consistent with $e_{i}$; it could however have become inconsistent with some previously treated edges. Nevertheless, after a sequence of $m$ steps all $m$ edges of $G$ have been treated once, and either the size of the current labeling decreases, or the labeling is consistent with every edge. Since the size of the initial labeling is $n k$, we are guaranteed, after at most $m n k$ steps, to obtain a labeling $l^{*}$ which is 
consistent with all edges; we call $l^{*}$ the standard consistent labeling of $G$ with respect to $H$, and say that the consistency check succeeds if $l^{*}(v) \neq \emptyset$ for all $v \in V(G)$.

Consistency checking goes back to early work on constraint satisfaction problems [29]. Its first use in homomorphism problems is due to Gutjahr et al. [15], to prove that the $H$-colouring problem is polynomial for any oriented path $H$. It was proved in [15], and it is also easy to see, that the standard consistent labeling $l^{*}$ is the maximum labeling, with respect to coordinate-wise inclusion, which is consistent with every edge; in other words, for any other labeling $l$ of $G$ with respect to $H$ which is consistent with respect to every edge of $G$, we have $l(x) \subseteq l^{*}(x)$ for all vertices $x$ of $G$.

If $G$ is homomorphic to $H$ then the consistency check for $G$ with respect to $H$ will succeed. Indeed for any homomorphism $h: G \rightarrow H$ each label $h(v)$ will remain a member of $l(v)$, in all steps of the consistency check. On the other hand, Lemma 2.1 below says that when $G$ is an oriented tree the success of the consistency check implies that $G \rightarrow H$. This is the essence of the folklore of arc consistency in the artificial intelligence community $[29,9]$. It also follows from the fact that, for any fixed $H$, the condition $G \rightarrow H$ can be expressed in monadic second-order logic $[1,8]$.

Our discussion will be facilitated by arguing about rooted homomorphisms: A rooted digraph $(G, g)$ is a digraph $G$ with a fixed vertex $g$ called the root. If $(G, g)$ and $\left(G^{\prime}, g^{\prime}\right)$ are rooted digraphs, then a rooted homomorphism $f:(G, g) \rightarrow\left(G^{\prime}, g^{\prime}\right)$ is a homomorphism $f: G \rightarrow G^{\prime}$ such that $f(g)=g^{\prime}$. We shall write $(G, g) \rightarrow\left(G^{\prime}, g^{\prime}\right)$ if such a homomorphism exists, and $(G, g) \nrightarrow\left(G^{\prime}, g^{\prime}\right)$ otherwise.

Lemma 2.1. Let $G$ be an oriented tree and $H$ any digraph, and suppose that the consistency check for $G$ with respect to $H$ succeeds. Then $G \rightarrow H$. Moreover, for any vertex $v$ of $G$ and any label $s$ of $l^{*}(v)$, we have $(G, v) \rightarrow(H, s)$.

Proof. Let $v \in V(G)$ and $s \in l^{*}(v)$. We will construct a mapping $h: V(G) \rightarrow V(H)$ as follows: First we set $h(v)=s$. For any $u$ with $v u \in E(G)$ there is a $t \in l^{*}(u)$ such that $s t \in E(H)$, because $l^{*}$ is consistent with the edge $v u$; we set $h(u)=t$. For any $w$ with $w v \in E(G)$ there is also a $z \in l^{*}(w)$ such that $z s \in E(H)$; we again set $h(w)=z$. This defines $h$ for all neighbours of $v$. Similarly we can extend the definition of $h$ to all neighbours of neighbours of $v$, and so on, until we have a mapping $h$ of $V(G)$ to $V(H)$. Since $G$ is an oriented tree, it is easy to see that such a mapping is indeed a homomorphism $h: G \rightarrow H$.

Corollary 2.2. Let $G$ and $H$ be any digraphs, and suppose that the consistency check for $G$ with respect to $H$ succeeds. If an oriented tree $T$ satisfies $T \rightarrow G$ then also $T \rightarrow H$.

Moreover, for any vertex $v$ of $G$ and any label $s \in l^{*}(v),(T, t) \rightarrow(G, v)$ implies $(T, t) \rightarrow(H, s)$, for any rooted oriented tree $(T, t)$.

Proof. Let $h:(T, t) \rightarrow(G, v)$ be a rooted homomorphism. Let $l_{1}^{*}$ be the standard consistent labeling of $G$, and let $l_{2}^{*}$ be the standard consistent labeling of $T$, both with respect to $H$. It is easy to see that $l_{1}^{*}(h(x))$ is a labeling of $T$ with respect to $H$ which is consistent with respect to each edge of $T$. Since $l_{2}^{*}$ is the maximum labeling consistent with each edge of $T$, we must have $l_{1}^{*}(h(x)) \subseteq l_{2}^{*}(x)$ for each $x \in V(T)$. Thus $s \in l_{1}^{*}(v) \subseteq l_{2}^{*}(t)$ and $(T, t) \rightarrow(H, s)$ by Lemma 2.1.

Theorem 2.3. Suppose $H$ has tree duality. Then the following three statements are equivalent: 
1. $G \rightarrow H$,

2. the consistency check for $G$ with respect to $H$ succeeds,

3. every tree homomorphic to $G$ is also homomorphic to $H$.

Proof. We have already observed that (1) implies (2) (before Lemma 2.1). Corollary 2.2 assures that (2) implies (3). Finally, (3) implies (1) since $H$ has tree duality.

Corollary 2.4. If $H$ has tree duality, then the $H$-colouring problem is polynomial.

Proof. The equivalence of the first two statements means we can use the consistency check.

To modify our technique to treewidth- $k$ duality we proceed as follows (assuming again $H$ is fixed): Instead of labeling the vertices of $G$ with sets of vertices of $H$, we shall label $k$-tuples of vertices of $G$ with sets of $k$-tuples of vertices of $H$. We say such a labeling $l$ is consistent with a $(k+1)$-element set $Y \subseteq V(G)$, if the following statement holds: Whenever $\left(x_{1}, x_{2}, \cdots, x_{k}\right)$ is a $k$-tuple of elements of $Y$ and $\left(r_{1}, r_{2}, \cdots, r_{k}\right) \in l\left(\left(x_{1}, x_{2}, \cdots, x_{k}\right)\right)$, there exists a homomorphism $h$ : $Y \rightarrow H$ with $h\left(x_{i}\right)=r_{i}$, for $i=1,2, \cdots, k$, such that $\left(h\left(x_{1}^{\prime}\right), h\left(x_{2}^{\prime}\right), \cdots, h\left(x_{k}^{\prime}\right)\right) \in$ $l\left(\left(x_{1}^{\prime}, x_{2}^{\prime}, \cdots, x_{k}^{\prime}\right)\right)$ for all $k$-tuples $\left(x_{1}^{\prime}, x_{2}^{\prime}, \cdots, x_{k}^{\prime}\right)$ of elements of $Y$. It is easy to see that in the case $k=1$, the labeling $l$ is consistent with a 2-element set $\left\{y_{1}, y_{2}\right\}$ if and only if it is consistent with the edge(s), if any, induced by $\left\{y_{1}, y_{2}\right\}$. We replace the consistency check by a $k$-consistency check: Instead of checking at each step an edge of $G$ (or equivalently, a pair of vertices of $G$ ) for consistency, we will check for consistency a $(k+1)$-set of vertices of $G$. Similarly, instead of modifying the current labeling to make it consistent with the chosen edge, we modify it to make it consistent with the chosen $(k+1)$-set. Thus we will obtain (in time polynomial in $|V(G)|)$ a labeling $l^{*}$ which is consistent with every $(k+1)$-set of vertices of $G$. We say that the $k$-consistency check succeeds if the final label $l^{*}$ of any $k$-tuple of vertices of $G$ is not the empty set.

It is again clear that if $G$ is homomorphic to $H$, then the $k$-consistency check will succeed. By an argument analogous to the above we also conclude that if the $k$-consistency check for $G$ succeeds, and $G$ has treewidth $k$, then $G$ is homomorphic to $H$. (This also follows from $[9,8]$.) Finally we can prove an analogue of Corollary 2.2 and the following theorem.

Theorem 2.5. Suppose $H$ has treewidth-k duality. Then the following three statements are equivalent:

1. $G \rightarrow H$,

2. the $k$-consistency check for $G$ with respect to $H$ will succeed,

3. every digraph of treewidth $k$ homomorphic to $G$ is also homomorphic to $H$.

Corollary 2.6. If $H$ has bounded treewidth duality, then the $H$-colouring problem is polynomial.

We shall now prove that the second and third statement of Theorems 2.3 and 2.5 are equivalent for all digraphs, cf. Theorem 2.8. Therefore $H$ has tree duality (treewidth- $k$ duality) if and only if the first and the second statements in Theorem 2.3 (respectively Theorem 2.5 ) are equivalent.

We begin with a lemma which is the converse of Lemma 2.2: 
Lemma 2.7. Let $l^{*}$ be the standard consistent labeling of $G$ with respect to $H$. For any vertex $x$ of $G$ and any vertex $r$ of $H$ such that $r \notin l^{*}(x)$, there is a rooted oriented tree $(T, t)$ which is homomorphic to $(G, v)$ but not to $(H, s)$.

Proof. Suppose to the contrary that there are $x \in V(G)$ and $r \in V(H)$ with $r \notin l^{*}(x)$, such that for every rooted oriented tree $(T, t)$ with $(T, t) \rightarrow(G, x)$, we have $(T, t) \rightarrow(H, r)$. The vertex $r$ was removed from $l(x)$ at a certain step, say at step $i$, in the process of computing $l^{*}$. We choose the pair $x, r$ so that the number $i$ is minimum, subject to the above assumption on $x$ and $r$. Assume without loss of generality that at step $i$, the edge being checked is $x y$. Thus there is no $s$ in $l(y)$ such that $r s \in E(H)$, where $l(y)$ is the label of $y$ at step $i$. For each $s \notin l(y)$ at step $i$, there is a rooted oriented tree $\left(T_{s}, t_{s}\right)$ such that $\left(T_{s}, t_{s}\right) \rightarrow(G, y)$ and $\left(T_{s}, t_{s}\right) \nrightarrow(H, s)$, for otherwise we would have chosen the pair $y, s$ instead of the pair $x, r$. We take all these rooted oriented trees $\left(T_{s}, t_{s}\right), s \notin l(y)$ at step $i$, and identify all roots $t_{s}$ to obtain a new tree $T$ with a vertex $t$ obtained from the identification. It is clear that for any homomorphism $h$ of $T$ to $H$ we have $h(t) \in l(y)$. Let $\left(T^{\prime}, t^{\prime}\right)$ be the rooted oriented tree obtained from $T$ by adding a new vertex $t^{\prime}$ and a new edge $t^{\prime} t$. It is easy to see that $\left(T^{\prime}, t^{\prime}\right) \rightarrow(G, x)$, which implies that $\left(T^{\prime}, t^{\prime}\right) \rightarrow(H, r)$ by our assumption. Thus there is a homomorphism $h^{\prime}$ of $T^{\prime}$ to $H$ such that $h^{\prime}\left(t^{\prime}\right)=r$. Let $s=h^{\prime}(t)$. Then $r s \in E(H)$ and $s \in l(y)$, where $l(y)$ is the label of $y$ at step $i$. This contradicts the assumption that there is no $s \in l(y)$ with $r s \in E(H)$.

Combining Lemma 2.1 and Lemma 2.7, we obtain the following result:

Theorem 2.8. Let $l^{*}$ be the standard consistent labeling of $G$ with respect to $H$, and let $v$ be a vertex of $G$. A vertex $r$ of $H$ is an element of $l^{*}(v)$ if and only if for every rooted oriented tree $(T, t)$ for which $(T, t) \rightarrow(G, v)$, we have $(T, t) \rightarrow(H, r)$.

Corollary 2.9. Tree duality of $H$ is equivalent to the following property: $G \rightarrow H$ if and only if the consistency check for $G$ with respect to $H$ succeeds.

For the standard $k$-consistent labeling $l^{*}$ there is an analogue to Theorem 2.8, with the appropriate definitions of homomorphisms of digraphs with $k$ specified vertices. (We write $\left(G, a_{1}, a_{2}, \ldots, a_{k}\right) \rightarrow\left(H, x_{1}, x_{2}, \ldots, x_{k}\right)$ when there is a homomorphism $f: G \rightarrow H$ with all $f\left(a_{i}\right)=x_{i}$.) In particular, treewidth- $k$ duality of $H$ is equivalent to the property that $G \rightarrow H$ if and only if the $k$-consistency check for $G$ with respect to $H$ succeeds.

Finally, we make another observation on the standard consistent labeling for balanced digraphs; this property will be needed in the next section. The proof is straightforward and will be omitted.

Lemma 2.10. Suppose $H$ is a balanced digraph. If $G$ is a digraph such that every tree homomorphic to $G$ is also homomorphic to $H$, then $G$ is also balanced and $h t(G) \leq h t(H)$. Moreover,

(i) if $h t(G)<h t(H)$ then there is a subgraph $H^{\prime}$ of $H$ of height $h t\left(H^{\prime}\right)=h t(G)$ such that every tree homomorphic to $G$ is also homomorphic to $H^{\prime}$, and

(ii) if $h t(G)=h t(H)$ then for any $x \in V(G)$, all vertices of $l^{*}(x)$ are on the same level. 


\section{Duality And the eXtended $\underline{X}$-PRoperty}

In [15] the authors define another property of digraphs which guarantees the existence of polynomial homomorphism algorithms. They call their property the extended $\underline{X}$-property, read ' $X$ underbar property'. (There is also a more special property simply called the $\underline{X}$-property.) This property implies the existence of many (but not all) known polynomial homomorphism algorithms, including that for oriented paths. We prove in this section that the class of digraphs with bounded treewidth duality (in fact with treewidth-2 duality) strictly contains the class of digraphs with the extended $\underline{X}$-property, cf. Theorem 3.1. Moreover, we do not know of any polynomial homomorphism algorithm which is not also implied by bounded treewidth duality. For balanced digraphs, even the class of digraphs with tree duality strictly contains the class of digraphs with the extended $\underline{X}$-property, cf. Theorem 3.2. (This is not so for unbalanced digraphs.) We also construct in this section several families of oriented trees that have the extended $\underline{X}$-property, and hence tree duality.

Let $H$ be a digraph and let $v_{1}, v_{2}, \cdots, v_{n}$ be an enumeration of the vertices of $H$. Two edges $v_{i} v_{j}$ and $v_{k} v_{l}$ are crossing if $i<k$ and $j>l$, or $i>k$ and $j<l$. The $\underline{X}$-pair of two crossing edges $v_{i} v_{j}$ and $v_{k} v_{l}$ is the ordered pair of vertices $v_{p} v_{q}$ where $p=\min \{i, k\}$ and $q=\min \{j, l\}$. Let $C_{m}$ denote the directed cycle with $m$ edges. A digraph $H$ has the extended $X$-property if there is a homomorphism $h: H \rightarrow C_{m}$, for some positive integer $m$, and an enumeration $v_{1}, v_{2}, \cdots, v_{n}$ of the vertices of $H$, such that for any two crossing edges $v_{i} v_{j}$ and $v_{k} v_{l}$ of $H$ with $h\left(v_{i}\right)=h\left(v_{k}\right)$, we have that the $\underline{X}$-pair of $v_{i} v_{j}$ and $v_{k} v_{l}$ is an edge of $H$. It is easy to see that a balanced digraph $H$ has the extended $X$-property if and only if there is an enumeration $v_{1}, v_{2}, \cdots, v_{n}$ of the vertices of $H$ such that for any two crossing edges $v_{i} v_{j}$ and $v_{k} v_{l}$ of $H$ with $v_{i}$ and $v_{k}$ on the same level, we have that the $\underline{X}$-pair of $v_{i} v_{j}$ and $v_{k} v_{l}$ is an edge of $H$.

Theorem 3.1. Any digraph $H$ which has the extended $X$-property also has treewidth-2 duality.

Proof. Let $v_{0}, v_{1}, \ldots, v_{n}$ be an enumeration of the vertices of $H$, and $h$ a homomorphism of $H$ to $C_{m}$, from the definition of the extended $X$-property. We shall show that $G \rightarrow H$ for any digraph $G$ for which the 2-consistency check (with respect to $H$ ) succeeds. We may assume that the underlying graph of $G$ is connected. Let $l^{*}$ be the standard 2-consistent labeling of $G$ with respect to $H$. Let $a$ be a fixed vertex of $G$. It follows from the 2-consistency of $l^{*}$ that there exists a vertex $x$ of $H$ with the following property: For each vertex $b$ of $G$ there is a vertex $y$ in $H$ such that $(x, y) \in l^{*}((a, b))$. Let $f(b)$ be the first such vertex $y$ in the above enumeration. Then we claim that $f$ is a homomorphism of $G$ to $H$. Suppose $b b^{\prime}$ is an edge of $G$ and $f(b)=y, f\left(b^{\prime}\right)=y^{\prime}$. Since $l^{*}$ is consistent, there exist vertices $z, z^{\prime}$ in $H$ such that $(x, z) \in l^{*}((a, b)),\left(z, y^{\prime}\right) \in l^{*}\left(\left(b, b^{\prime}\right)\right)$, and $\left(x, z^{\prime}\right) \in l^{*}\left(\left(a, b^{\prime}\right)\right),\left(y, z^{\prime}\right) \in l^{*}\left(\left(b, b^{\prime}\right)\right)$. By the minimality of $y, y^{\prime}$ we conclude that $z y^{\prime}, y z^{\prime}$ are two crossing edges. We now show that $h(y)=h(z)$ : Let $P$ be any oriented path (say, of algebraic length $l$ ) joining $a$ and $b$. Since $(P, a, b) \rightarrow(G, a, b)$, we also have $(P, a, b) \rightarrow(H, x, y)$ (this follows from $(x, y) \in l^{*}((a, b))$ and the analogue of Theorem 2.8). The image of $P$ in $H$ under this homomorphism is an oriented walk joining $x$ and $y$, of algebraic length $l$. Similarly, there is in $H$ an oriented walk of algebraic length $l+1$ joining $x$ and $y^{\prime}$ (which is the image of the concatenation of $P$ and the edge $b b^{\prime}$ ). This implies 
that $H$ contains an oriented walk of algebraic length $l$ joining $x$ and $z$, since $z y^{\prime}$ is an edge of $H$. Therefore $z$ and $y$ have the same image under any homomorphism of $H$ to a directed cycle. Thus $h(y)=h(z)$ and the $\underline{X}$-pair of $z y^{\prime}, y z^{\prime}$, namely $y y^{\prime}$, is an edge of $H$.

We note that in the above theorem we cannot replace treewidth-2 duality with tree duality. Directed cycles have the extended $\underline{X}$-property and do not have tree duality (every oriented tree is homomorphic to a directed cycle). On the other hand we prove:

Theorem 3.2. Any balanced digraph (and hence any oriented tree) $H$ which has the extended $\underline{X}$-property also has tree duality.

Proof. Suppose that there exist balanced digraphs that have the extended $X$ property but do not have tree duality, and let $H$ be such a digraph with the smallest number of vertices. Let $v_{1}, v_{2}, \cdots, v_{n}$ be an enumeration of the vertices of $H$ from the equivalent definition of the extended $\underline{X}$-property for balanced digraphs. Let $G$ be a digraph which is not homomorphic to $H$ and such that every oriented tree homomorphic to $G$ is also homomorphic to $H$. By Lemma 2.7, the standard consistent labeling $l^{*}$ of $G$ with respect to $H$ satisfies $l^{*}(x) \neq \emptyset$ for all $x \in V(G)$. By Lemma 2.10, $G$ is balanced and $h t(G) \leq h t(H)$. Moreover, if $h t(G)<h t(H)$ then there is a subgraph $H^{\prime}$ of $H$ with $h t\left(H^{\prime}\right)=h t(G)$ such that every oriented tree homomorphic to $G$ is also homomorphic to $H^{\prime}$. Since $H^{\prime}$ also has the extended $\underline{X}$-property, we know that $H^{\prime}$ has tree duality by the minimality of $H$. Thus $G$ is homomorphic to $H^{\prime}$, and hence to $H$, contrary to our assumption.

Thus we may assume that $h t(G)=h t(H)$. Let $h(x)$ be the first element of $l^{*}(x)$ in the enumeration $v_{1}, v_{2}, \cdots, v_{n}$. We now show that $h$ is a homomorphism of $G$ to $H$, contrary to our assumption. Suppose $x y$ is an edge of $G$ and $h(x)=v_{i}, h(y)=v_{l}$. We shall show that $v_{i} v_{l}$ is an edge of $H$. Since $l^{*}$ is consistent with the edge $x y$, there is $v_{j} \in l^{*}(y)$ and $v_{k} \in l^{*}(x)$ such that $v_{i} v_{j}, v_{k} v_{l}$ are edges of $H$. If $i=k$ or $j=l$ then $h(x) h(y)$ is an edge of $H$ and we are done. Otherwise by the definition of $f, i<k$ and $j>l$, i.e., they are crossing edges. Since $d_{H}\left(v_{i}, v_{k}\right)=0$ by Lemma 2.10 , and $H$ has the extended $\underline{X}$-property, the $\underline{X}$-pair of these two edges, which is $v_{i} v_{l}$, must be an edge of $H$.

In the rest of this section we present some families of oriented trees that have the extended $\underline{X}$-property, and hence tree duality. We recursively define the class $\mathcal{T}$ of rooted oriented trees as follows:

- Suppose $P$ is an oriented path with one end-vertex $v$; if all other vertices $u$ of $P$ have $d_{P}(v, u) \neq 0$, then $(P, v) \in \mathcal{T}$.

- Let $V(T)=\left\{v_{1}, v_{2}, \ldots, v_{n}\right\}$. Suppose that $(T, v) \in \mathcal{T}$ and $\left(T_{i}, u_{i}\right) \in \mathcal{T}$, for each $i=1,2, \ldots, n$. Let $T^{\prime}$ be the oriented tree obtained from the disjoint union of $T$ and $T_{i}$ by identifying the vertex $v_{i}$ of $T$ with the vertex $u_{i}$ of $T_{i}$, for each $i=1,2, \cdots, n$. (We call this operation planting.) If all other vertices $u$ of $T^{\prime}$ have $d_{T}(v, u) \neq 0$, then $\left(T^{\prime}, v\right) \in \mathcal{T}$.

Theorem 3.3. Every rooted oriented tree $(T, v)$ from the class $\mathcal{T}$ has the extended $\underline{X}$-property with respect to some enumeration of $T$ starting at the root $v$.

Proof. We prove this theorem by induction on the number of vertices of $T$. If $|V(T)|=1$ then the theorem is obviously true. Now suppose that the theorem is true for any $\left(T^{\prime}, v^{\prime}\right) \in \mathcal{T}$ with at most $n-1$ vertices, and suppose that $(T, v) \in \mathcal{T}$ 
has $n$ vertices. If $T$ is an oriented path and $v$ is an end vertex of $T$, then we take the natural enumeration of the oriented path starting at the end vertex $v$. It was proved

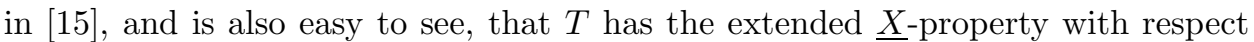
to this enumeration. (In fact, it has the more restrictive $X$-property [15].) Now suppose that $(T, v)$ is obtained from some $\left(T_{0}, v\right) \in \mathcal{T}$ by planting at each vertex $v_{i}$ of $T_{0}$ a rooted tree $\left(T_{i}, u_{i}\right) \in \mathcal{T}$. Clearly we can assume that $T_{0}$ and each $T_{i}$ has at most $n-1$ vertices. Thus by the induction hypothesis, $\left(T_{0}, v\right)$, and each of the planted trees $\left(T_{i}, u_{i}\right)$, has a required enumeration. Without loss of generality, we assume that the enumeration of $T_{0}$ is $v=v_{1}, v_{2}, \ldots, v_{m}$. Then the enumeration of $(T, v)$ is the concatenation of the enumerations of $\left(T_{1}, u_{1}\right),\left(T_{2}, u_{2}\right), \ldots,\left(T_{m}, u_{m}\right)$. Assume that this enumeration is $w_{1}, w_{2}, \cdots, w_{n}$. It starts at the root $v=w_{1}=v_{1}=u_{1}$. To see that $T$ has the extended $\underline{X}$-property with respect to this enumeration, we take two crossing edges $w_{i} w_{j}$ and $w_{k} w_{l}$ such that $w_{i}$ and $w_{k}$ are on the same level. Without loss of generality, we assume that $i<k$ and $j>l$. If these two edges belong to one of the trees $\left(T_{q}, u_{q}\right)$, then the $\underline{X}$-pair of $\left(w_{i}, w_{j}\right)$ and $\left(w_{k}, w_{l}\right)$ is an edge of $T_{q}$ and hence an edge of $T$. Suppose $w_{i} w_{j}$ and $w_{k} w_{l}$ belong to different subtrees, say $w_{i} w_{j}$ is an edge of $T_{p}, w_{k} w_{l}$ is an edge of $T_{q}$. Consider the case that $p<q$. (The case $p>q$ can be treated similarly.) Since $i<k$ and $j>l$, we must have $p=0$, and $T_{q}$ must be the tree planted at $w_{i}=v_{q}$. By assumption $d_{T_{q}}\left(v_{q}, u\right) \neq 0$ for all other vertices of $T_{q}$. Hence $d_{T}\left(w_{i}, w_{k}\right) \neq 0$, contrary to the assumption that $w_{i}$ and $w_{k}$ are on the same level. Therefore the tree $T$ has the extended $\underline{X}$-property with respect to this enumeration.

Corollary 3.4. Let $T$ be an oriented tree with vertices $v_{1}, v_{2}, \cdots, v_{n}$. Suppose that $T$ has the extended $\underline{X}$-property and that $\left(T_{i}, u_{i}\right), i=1,2, \ldots, n$, are elements of $\mathcal{T}$. Then the tree $T^{\prime}$ obtained from $T$ by planting at each $v_{i}$ the rooted oriented tree $\left(T_{i}, u_{i}\right)$ also has the extended $\underline{X}$-property.

Proof. Suppose $T$ has the extended $\underline{X}$-property with respect to the enumeration $v_{1}, v_{2}, \cdots, v_{n}$. By Theorem 3.3, each of the trees $\left(T_{i}, u_{i}\right)$ has the extended $\underline{X}$ property with respect to an enumeration starting at $u_{i}$. We take the concatenation of the enumerations of $\left(T_{1}, u_{1}\right),\left(T_{2}, u_{2}\right), \cdots,\left(T_{n}, u_{n}\right)$, and obtain an enumeration of the vertices of $T^{\prime}$. The same argument as in the proof of Theorem 3.3 shows that $T^{\prime}$ has the extended $\underline{X}$-property with respect to this enumeration.

We close this section with another family of oriented trees which have the extended $\underline{X}$-property. It is a family of oriented triads. Formally, $T$ is an oriented triad if the underlying undirected tree of $T$ has exactly one vertex $v$ of degree three and all other vertices of degree one or two. We view $T$ as consisting of three oriented paths $W_{1}, W_{2}$, and $W_{3}$ starting at the unique vertex $v$ of degree three and ending at vertices $z_{1}, z_{2}, z_{3}$.

Theorem 3.5. Let $T$ be an oriented triad with paths $W_{1}, W_{2}, W_{3}$, meeting at $v$ as above. Suppose there exists a vertex $t$ on the path $W_{1}$, such that the path joining $v$ and $t$ is minimal, and such that no vertex of $W_{2}$ is on the same level as $t$. Then $T$ has the extended $\underline{X}$-property.

Proof. We again give an enumeration $a_{1}, a_{2}, \ldots, a_{l}$ of the vertices of $T$, from the equivalent definition of the extended $\underline{X}$-property for balanced digraphs. Let $u$ be the first vertex after $v$ on $W_{2}$, and $w$ the first vertex after $t$ on the path from $t$ to $z_{1}$. We enumerate the vertices of $T$ as follows: First enumerate the vertices of $W_{3}$ 
from $z_{3}$ to $v$, then enumerate the vertices of $W_{1}$ from $v$ up to $t$, then enumerate the vertices of $W_{2}$ from $u$ to $z_{2}$, and finally enumerate the vertices of $W_{1}$ from $w$ to $z_{1}$. We now verify that $T$ has the extended $\underline{X}$-property with respect to this enumeration. Let $a_{i} a_{j}$ and $a_{k} a_{l}$ be a pair of crossing edges with $a_{i}$ and $a_{k}$ on the same level. We assume that $i<k$ and $j>l$. From the enumeration of the vertices of $T$ it is easy to see that either $a_{k}=w, a_{l}=t$ and $a_{i} a_{j}$ is an edge of $W_{2}$, or $a_{i}=v, a_{j}=u$ and $a_{k} a_{l}$ is an edge of the path joining $v$ and $t$. In the former case $a_{i}$ would be a vertex on $W_{2}$ on the same level as $t$ contradicting our assumption. In the latter case, $a_{k}$ and $v$ would be on the same level, contradicting the minimality of the path joining $v$ and $t$.

\section{Special triads}

Oriented triads are the "simplest" oriented trees other than oriented paths. As we have remarked above the $H$-colouring problem for any oriented path $H$ is polynomial [15, 23]. However, there exist oriented triads $H$ with $N P$-complete $H$-colouring problems, Theorem 4.1. (The smallest oriented triad to which this theorem applies has 45 vertices.) In order to state our results in the simplest possible form (although some of the results hold more generally) we concentrate in this section on a particular class of oriented triads. We say that the oriented triad $T$ is a special triad if there exist minimal oriented paths $P_{1}, P_{2}, \ldots, P_{6}$ of the same algebraic length (say al $\left(P_{i}\right)=n$, for all $\left.i=1,2, \ldots, 6\right)$, such that $W_{1}$ is the concatenation of $P_{1}$ and $P_{4}^{-1}, W_{2}$ is the concatenation of $P_{2}$ and $P_{5}^{-1}$, and $W_{3}$ is the concatenation of $P_{3}$ and $P_{6}^{-1}$. We denote by $t_{i}$ the vertex of $W_{i}$ which is common to $P_{i}$ and $P_{i+3}^{-1}$.

Theorem 4.1. [20] Let $T$ be a special triad. Suppose that

- for any $i, j \in\{1,2,3\}$, there is a minimal oriented path $P_{i j}$ of net length $n$ which is homomorphic to $P_{i}$ and $P_{j}$, but not homomorphic to $P_{k}$ for any $k=1,2, \ldots, 6, k \neq i, j$, and

- for any $i \in\{1,2,3\}$, there is a minimal oriented path $P_{i}^{*}$ of net length $n$ such that $P_{i}^{*}$ is homomorphic to $P_{i}, P_{4}, P_{5}$ and $P_{6}$, but not homomorphic to $P_{k}$ for any $k=1,2,3, k \neq i$.

Then the T-colouring problem is NP-complete.

Here we contrast these with several classes of special triads $H$ which have polynomial $H$-colouring problems. (Some of these are strikingly similar to the above triads with $N P$-complete problems.) We construct special triads which have tree

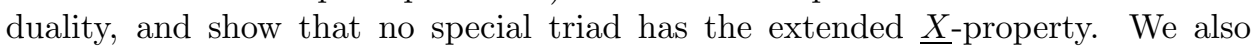
construct special triads which have treewidth-2 duality, some of which do not have tree duality. (We do not know if there exist oriented trees - or any digraphs - which have bounded treewidth duality but do not have treewidth-2 duality.)

First we observe that no special triad has the extended $X$-property. Suppose, to the contrary, that there is a special triad $T$, with vertices named as in Figure 1 , which has the extended $\underline{X}$-property. Let $a_{1}, a_{2}, \cdots, a_{l}$ be an enumeration of the vertices of $T$ from the equivalent definition of the extended $X$-property for balanced digraphs. Then there are two vertices from $z_{1}, z_{2}, z_{3}$ which both precede $v$ or both follow $v$ in the enumeration. Without loss of generality, let both $z_{1}$ and $z_{2}$ precede $v$. We write $a \leq b$ if $a=b$ or $a$ precedes $b$ in the above enumeration. Since $v, z_{1}$ are

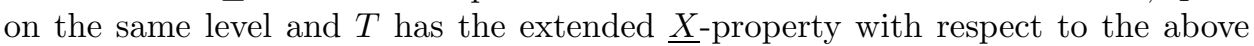
enumeration, we conclude that $x \leq y$ for any $x$ and $y$ on the same level for which 
$x$ is adjacent to $z_{1}$ and $y$ is adjacent to $v$. Similarly, we conclude that $x \leq y$ for any $x$ and $y$ on the same level for which $x$ is adjacent to $z_{2}$ and $y$ is adjacent to $v$. Repeating this argument, we see that $x \leq y$ for any vertex $x \in P_{1} \cup P_{2}$ and any vertex $y \in P_{4} \cup P_{5}$, such that $x, y$ are on the same level. This implies that $t_{1} \leq t_{2}$ and $t_{2} \leq t_{1}$, which is a contradiction. Our next theorem gives a family of special triads with tree duality.

Theorem 4.2. Suppose that $T$ is a special triad. If $P_{1} \rightarrow P_{2}$ then $T$ has tree duality.

Proof. Suppose that the theorem is not true and that $T$ is a special triad which satisfies the condition of the theorem but does not have tree duality. Let $G$ be a digraph such that every tree homomorphic to $G$ is also homomorphic to $T$, and yet $G$ is not homomorphic to $T$. By Lemma $2.10, G$ is balanced and $h t(G) \leq h t(T)$. If the height $h t(G)$ of $G$ is smaller than the height of $T$, then there is a subtree $T^{\prime}$ of $T$ such that any tree homomorphic to $G$ is also homomorphic to $T^{\prime}$. It is easy to verify that any proper subtree $T^{\prime}$ of $T$ satisfies the condition of Corollary 3.4 and hence has tree duality. Therefore $G$ is homomorphic to $T^{\prime}$, and hence homomorphic to $T$, which is a contradiction. Thus $h t(G)=h t(T)$. In this case we shall also construct a homomorphism of $G$ to $T$, and so obtain a final contradiction.

We denote by $v_{1}, v_{2}, v_{3}$ the vertices of $P_{1}, P_{2}, P_{3}$ respectively, which are adjacent to $v$ (the unique vertex of $T$ with degree three).

Lemma 4.3. Suppose that $x y$ is an edge of $G$. If $v \in l^{*}(x), v_{2} \notin l^{*}(y)$ and $v_{3} \notin l^{*}(y)$, then $l^{*}(x) \cap V\left(W_{2}\right)=\{v\}$.

Proof. Since $v \in l^{*}(x)$, and the only other vertex in $W_{2}$ on the same level as $v$ is $z_{2}$, we have $l^{*}(x) \cap V\left(W_{2}\right) \subseteq\left\{v, z_{2}\right\}$. We shall prove that $z_{2} \notin l^{*}(x)$. Suppose to the contrary that $z_{2} \in l^{*}(x)$.

Since $v_{2} \notin l^{*}(y)$ and $v_{3} \notin l^{*}(y)$, there is a rooted oriented tree $\left(T^{\prime}, b\right)$ such that $\left(T^{\prime}, b\right) \rightarrow(G, y)$ and $\left(T^{\prime}, b\right)$ is not homomorphic to either $\left(T, v_{2}\right)$ or $\left(T, v_{3}\right)$. Add to $T^{\prime}$ a vertex $a$ and the edge $a b$, to obtain another rooted oriented tree $\left(T^{\prime \prime}, a\right)$. Obviously $\left(T^{\prime \prime}, a\right) \rightarrow(G, x)$. Since $v \in l^{*}(x)$, there is a homomorphism $f$ : $\left(T^{\prime \prime}, a\right) \rightarrow(T, v)$. Now $f(b)$ is adjacent to $v$, and $f(b) \neq v_{2}, v_{3}$. Therefore $f(b)=v_{1}$. Since $z_{2} \in l^{*}(x)$, there is a homomorphism $f^{\prime}:\left(T^{\prime \prime}, a\right) \rightarrow\left(T, z_{2}\right)$. We shall define a new homomorphism $f^{\prime \prime}$ by combining $f$ and $f^{\prime}$ so that $f^{\prime \prime}(b)=v_{2}$, which will then contradict the assumption that $\left(T^{\prime}, b\right)$ is not homomorphic to $\left(T, v_{2}\right)$.

For any vertex $z$ of $T^{\prime \prime}$, let $P_{z}$ be the path of $T^{\prime \prime}$ connecting $z$ and $a$. Let $X$ be the set of vertices $z$ such that $P_{z}$ is a minimal path of algebraic length $n$. Let $Y$ be the set of vertices $z$ such that $a l\left(P_{z}\right)=0$ and $P_{z}$ contains no other vertex $s$ with $a l\left(P_{s}\right)=0$ or $n$. Let $k$ be a homomorphism of $P_{1}$ to $P_{2}$. It is easily verified that the homomorphisms $k \circ f$ and $f^{\prime}$ agree on the set $X$, and the homomorphisms $k \circ f$ and $f$ agree on the set $Y$. For example, if $z \in X$ then $f\left(P_{z}\right)$ must be a minimal path of algebraic length $n$. This implies that $f\left(P_{z}\right)=P_{1}$, because $f(b)=v_{1}\left(b \in P_{z}\right)$ and $P_{1}$ is the unique minimal path of $T$ containing $v_{1}$. Therefore $f(z)=t_{1}$ and hence $k(f(z))=t_{2}$. Similarly $f^{\prime}\left(P_{z}\right)$ is a minimal path of algebraic length $n$ containing $z_{2}$. Thus $f^{\prime}\left(P_{z}\right)=P_{5}$ and $f^{\prime}(z)=t_{2}$. Analogously, we can show that for each $z \in Y, k(f(z))=f(z)=v$.

Let $A$ be the component of $T^{\prime \prime} \backslash(X \cup Y)$ which contains $a$ (and hence $b$ ), let $B$ be the set of vertices $z$ such that $P_{z}$ contains a vertex of $X$, and let $C$ be the set of vertices $z$ such that $P_{z}$ contains a vertex of $Y$. Then $\{A, B, C\}$ is a partition 
of the vertex set of $T$. Since the homomorphisms $k \circ f$ and $f^{\prime}$ agree on $X$, and the homomorphisms $k \circ f$ and $f$ agree on $Y$, the mapping $f^{\prime \prime}: T^{\prime \prime} \rightarrow T$ defined by $f^{\prime \prime}(z)=k(f(z))$ for $z \in A, f^{\prime \prime}(z)=f^{\prime}(z)$ for $z \in B$, and $f^{\prime \prime}(z)=f(z)$ for $z \in C$, is a homomorphism of $T^{\prime \prime}$ to $T$. It is easily seen from the definition that $f(b)=v_{2}$. This contradicts the assumption that $\left(T^{\prime \prime}, b\right) \nrightarrow\left(T, v_{2}\right)$.

Continuing with the proof of Theorem 4.2, we proceed to construct a homomorphism of $G$ to $T$. First we define an enumeration $a_{1}, a_{2}, \ldots, a_{l}$ of the vertices of $T$ : First enumerate the vertices of $W_{3}$ from $z_{3}$ to $t_{3}$, then the vertices of $W_{2}$ from $v$ to $z_{2}$, and finally the vertices of $W_{1}$ from $v_{1}$ to $z_{1}$. Suppose that $v=a_{k}$ and $v_{1}=a_{r}$. We let $f(x)$ be the first element of $l^{*}(x)$ in the enumeration $a_{1}, a_{2}, \ldots, a_{l}$. We now show that $f$ is a homomorphism. Let $x y$ be an edge of $G$ and suppose that $f(x)=a_{i}$ and $f(y)=a_{j}$. Since $l^{*}$ is consistent, there is an $a_{q} \in l^{*}(y)$ such that $a_{i} a_{q}$ is an edge of $T$, and an $a_{p} \in l^{*}(x)$ such that $a_{p} a_{j}$ is an edge of $T$. If $p=i$ or $j=q$ then we are done. Otherwise we have $i<p$ and $j<q$. From the enumeration of the vertices of $T$ we see that this is possible only if one of the edges $a_{i} a_{q}$ and $a_{p} a_{j}$ is the edge $v v_{1}$ and the other is an edge of $W_{2} \backslash\{v\}$. This implies, in particular, that $v \in l^{*}(x)$ and $\left(l^{*}(x) \cap V\left(W_{2}\right)\right) \backslash\{v\} \neq \emptyset$. By Lemma 4.3, $v_{2} \in l^{*}(y)$ or $v_{3} \in l^{*}(y)$. However $v_{3}$ and $v_{2}$ precede any vertex of $W_{2} \backslash\{v\}$ in the enumeration, and in particular precede $a_{j}$, which is the first element of $l^{*}(y)$. Therefore $v_{2}=a_{j}$ and hence $a_{i} a_{j}$ is an edge of $T$.

Let $T$ be a special triad; we say that $q:\{4,5,6\} \rightarrow\{1,2,3\}$ is a compatible mapping of $T$, if $P_{i} \rightarrow P_{q(i)}$ for $i=1,2,3$. We show below (Theorem 4.4) that a special triad which admits a compatible mapping has treewidth-2 duality. We first point out that there are special triads which admit a compatible mapping, yet do not have tree duality. Let $P_{1}, P_{2}, P_{3}$ be minimal oriented paths of algebraic length $n$ such that there is no homomorphism from $P_{i}$ to $P_{j}$ for any $i \neq j$, and suppose that there exist oriented paths $P_{12}, P_{13}, P_{23}$ with each $P_{i j}$ homomorphic to $P_{i}$ and $P_{j}$ but to no other $P_{k}$. Such paths $P_{1}, P_{2}, P_{3}$ are easily constructed, when $n \geq 4$. Let $T$ be the special triad with $P_{1}, P_{2}, P_{3}, P_{4}=P_{2}, P_{5}=P_{3}, P_{6}=P_{1}$. Clearly, $T$ is a core and admits a compatible mapping. We now argue that $T$ does not have tree duality. Let $P$ be the oriented path obtained by concatenating $P_{13}^{-1}, P_{3}, P_{12}^{-1}, P_{1}, P_{23}^{-1}$, and $P_{2}$. Let $C$ be the oriented cycle obtained from $P$ by identifying the two end vertices of $P$. Then the resulting oriented cycle $C$ is easily seen to not be homomorphic to $T$, yet every oriented tree homomorphic to $C$ is also homomorphic to $T$.

Theorem 4.4 was first proved (for the special case described above where $P_{4}=$ $\left.P_{2}, P_{5}=P_{3}, P_{6}=P_{1}\right)$ by $\mathrm{T}$. Feder, personal communication.

Theorem 4.4. Every special triad which admits a compatible mapping has treewidth-2 duality.

Proof. The proof is based on a technique of Feder and Vardi [11], which uses the existence of a certain homomorphism $g$ to prove that if the 2-consistency check for $G$ with respect to $T$ succeeds, then there is a homomorphism of $G$ to $T$. This implies in particular that if each oriented partial 2-tree homomorphic to $G$ is also homomorphic to $T$, then (the 2-consistency check for $G$ with respect to $T$ succeeds and) $G \rightarrow T$. Thus the existence of such a homomorphism $g$ is stronger than treewidth-2 duality; we refer the reader to [11] for other interesting applications of this concept. Specifically, let $T^{*}$ be the digraph with vertices $(x, y, z) \in V(T) \times V(T) \times V(T)$ such that $x, y, z$ are on the same level in $T$, and in which $(x, y, z)\left(x^{\prime}, y^{\prime}, z^{\prime}\right)$ is an 
edge exactly when $x x^{\prime}, y y^{\prime}, z z^{\prime}$ are edges in $T$. Suppose there is a homomorphism $g: T^{*} \rightarrow T$ such that $g(x, y, z)=a$ if at least two of $x, y, z$ are equal to $a$. We now claim that if the 2-consistency check for $G$ with respect to $T$ succeeds then $G \rightarrow T$. Note that the success of the 2-consistency check implies that $G$ is balanced, of height at most $h t(T)$. By using Lemma 2.10, we only need to consider the case $h t(G)=h t(T)$. This implies, by Lemma 2.10, that for a pair $\left(x, x^{\prime}\right)$ of vertices of $G$, if $\left(y_{1}, y_{1}^{\prime}\right),\left(y_{2}, y_{2}^{\prime}\right) \in l^{*}\left(\left(x, x^{\prime}\right)\right)$ then $y_{1}, y_{2}$ are on the same level, and $y_{1}^{\prime}, y_{2}^{\prime}$ are also on the same level.

First we show that for any pair $\left(x, x^{\prime}\right)$ of vertices of $G$, if $\left(y_{1}, y_{1}^{\prime}\right),\left(y_{2}, y_{2}^{\prime}\right)$, $\left(y_{3}, y_{3}^{\prime}\right) \in l^{*}\left(\left(x, x^{\prime}\right)\right)$, and if we let $g=g\left(y_{1}, y_{2}, y_{3}\right), g^{\prime}=g\left(y_{1}^{\prime}, y_{2}^{\prime}, y_{3}^{\prime}\right)$, then $\left(g, g^{\prime}\right) \in$ $l^{*}\left(\left(x, x^{\prime}\right)\right)$. By the analogue of Corollary 2.9 , it suffices to show that for any partial 2 -tree $F$ and any two specified vertices $t, t^{\prime}$, with $\left(F, t, t^{\prime}\right) \rightarrow\left(G, x, x^{\prime}\right)$, we also have $\left(F, t, t^{\prime}\right) \rightarrow\left(T, g, g^{\prime}\right)$. Let $\left(F, t, t^{\prime}\right)$ be a partial 2-tree with two specified vertices such that $\left(F, t, t^{\prime}\right) \rightarrow\left(G, x, x^{\prime}\right)$. Since $\left(y_{1}, y_{1}^{\prime}\right),\left(y_{2}, y_{2}^{\prime}\right),\left(y_{3}, y_{3}^{\prime}\right) \in l^{*}\left(\left(x, x^{\prime}\right)\right)$, there exist homomorphisms $h_{1}:\left(F, t, t^{\prime}\right) \rightarrow\left(T, y_{1}, y_{1}^{\prime}\right), h_{2}:\left(F, t, t^{\prime}\right) \rightarrow\left(T, y_{2}, y_{2}^{\prime}\right)$, and $h_{3}:\left(F, t, t^{\prime}\right) \rightarrow\left(T, y_{3}, y_{3}^{\prime}\right)$. If $h: F \rightarrow T$ is defined as $h(v)=g\left(h_{1}(v), h_{2}(v), h_{3}(v)\right)$, then it is easily seen that $h$ is a homomorphism of $\left(F, t, t^{\prime}\right)$ to $\left(T, g, g^{\prime}\right)$.

We say that a homomorphism $h$ of an induced subgraph of $G$ to $T$ is safe if $(h(u), h(v)) \in l^{*}((u, v))$ for all vertices $u, v$ of the subgraph. We now prove, by induction on $k$, that for every set $X=\left\{x_{1}, x_{2}, \ldots, x_{k}\right\}$ of vertices of $G$, every safe homomorphism $h$ of the subgraph of $G$ induced by $X$ to $T$, and every $x \in V(G)-X$, there is a safe homomorphism of the subgraph of $G$ induced by $X \cup x$ to $T$ which extends $h$. By the consistency of $l^{*}$, this is true if $k \leq 2$. Assume now that $k \geq 3$. Let $h_{i}, i=1,2,3$, be safe extensions of $h$ restricted to $X-x_{i}$ from $X-x_{i}$ to $\left(X-x_{i}\right) \cup x$ (which exist by the induction hypothesis). Then we claim that extending $h$ from $X$ to $X \cup x$ by setting $h(x)=g\left(h_{1}(x), h_{2}(x), h_{3}(x)\right)$ yields a safe homomorphism to $T$. According to the definition of a 2-consistent labeling, it will be enough to verify that for any $x_{i},\left(h(x), h\left(x_{i}\right)\right) \in l^{*}\left(\left(x, x_{i}\right)\right)$ (this will also imply that $h$ preserves edges). At least two of the three homomorphisms $h_{1}, h_{2}, h_{3}$ are defined on both $x$ and $x_{i}$. Without loss of generality, assume that this is so for $h_{1}, h_{2}$. Then we have $\left(h_{1}(x), h\left(x_{i}\right)\right) \in l^{*}\left(\left(x, x_{i}\right)\right)$ and $\left(h_{2}(x), h\left(x_{i}\right)\right) \in l^{*}\left(\left(x, x_{i}\right)\right)$. Let $z$ be any vertex of $T$ such that $\left(h_{3}(x), z\right) \in l^{*}\left(\left(x, x_{i}\right)\right)$ (such a vertex exists as $l^{*}$ is consistent). Since $g\left(h_{1}(x), h_{2}(x), h_{3}(x)\right)=h(x)$ and $g\left(h\left(x_{i}\right), h\left(x_{i}\right), z\right)=h\left(x_{i}\right)$, we have $\left(h(x), h\left(x_{i}\right)\right) \in l^{*}\left(\left(x, x_{i}\right)\right)$, by the previous paragraph. It is easy to see that the 2-consistency of $l^{*}$ implies that $h$ is a homomorphism.

It remains to show that such a homomorphism $g: T^{*} \rightarrow T$ exists. For each $j=4,5,6$, there is a homomorphism $\phi_{j}: P_{j} \rightarrow P_{q(j)}$. Without loss of generality, we can assume that either $q(4), q(5), q(6)$ are all distinct or at least two of them equal 1. Let $\phi$ be the mapping of $V(T)$ to $V(T)$ defined as $\phi(r)=\phi_{j}(r)$, if $r \in P_{j}$ with $j \in\{4,5,6\}$, and $\phi(r)=r$ otherwise. We now define the mapping $g(x, y, z)$ as follows: If all three vertices $x, y, z$, lie on one path of $T$, then $g(x, y, z)$ is the middle vertex of the three. (This means in particular that $g(x, y, z)=a$ if two of $x, y, z$ are equal to $a$.) If each of the three vertices $x, y, z$ lies on a different path from the paths $P_{1}, P_{2}, P_{3}$, then $g(x, y, z)$ is that vertex from $x, y, z$ which lies on $P_{1}$. For all the other triples $x, y, z$, it is easy to see that the triple $\phi(x), \phi(y), \phi(z)$ must fall into one of the two cases above; we define $g(x, y, z)=g(\phi(x), \phi(y), \phi(z))$. It is a routine (although tedious) exercise to check that $g$ is indeed a homomorphism $T^{*} \rightarrow T$. 
In view of Theorem 2.5, the special triads $H$ from Theorem 4.1 cannot have bounded treewidth duality, unless $P=N P$. It would be interesting to supply a proof of this, without using the assumption that $P \neq N P$. The next result is a first step in this direction.

Theorem 4.5. Let $T$ be any special triad from Theorem 4.1. Then $T$ does not have tree duality.

Proof. Let $G$ be the digraph depicted in Figure 2, where the paths $P_{i}^{*}, P_{i j}$ are defined as in Theorem 4.1. We claim that $G$ is not homomorphic to $T$, yet every tree homomorphic to $G$ is homomorphic to $T$.

Assume first that there is a homomorphism $h: G \rightarrow T$. It is easy to see that for $i=2,4,6, h\left(x_{i}\right)$ is equal to either $v$ or $u_{j}$ for some $j \in\{1,2,3\}$. At most one of $h\left(x_{2}\right), h\left(x_{4}\right), h\left(x_{6}\right)$ can be equal to $v$. (If, say $h\left(x_{2}\right)=h\left(x_{4}\right)=v$, then $h\left(x_{3}\right)$ would have to be equal to both $t_{2}$ and $t_{3}$.) Thus without loss of generality assume that $h\left(x_{2}\right)=u_{i}$ and $h\left(x_{4}\right)=u_{j}$, for some $i, j \in\{1,2,3\}$. If $i \neq j$ or if $i=j=1$ then again we obtain a contradiction with the value of $h\left(x_{3}\right)$. If $i=j=2$, then the value $h\left(x_{5}\right)$ yields a contradiction; and if $i=j=3$, then there is a similar contradiction for $h\left(x_{1}\right)$. Therefore $G$ is not homomorphic to $T$.

We now show that every tree homomorphic to $G$ is also homomorphic to $T$. Let $X$ be the tree obtained from $G$ by spliting $x_{6}$ into two vertices $x_{6}^{\prime}$ and $x_{6}^{\prime \prime}$. Take infinitely many copies of $X$, say $X_{1}, X_{2}, X_{3}, \cdots$, and identify the vertex $x_{6}^{\prime}$ of each $X_{i}$ with the vertex $x_{6}^{\prime \prime}$ of $X_{i+1}$, obtaining an infinite tree $T^{\prime}$. It is a routine excercise to verify that $T^{\prime} \rightarrow T$ (the consecutive vertices corresponding to $x_{6}$ alternately map to $u_{1}$ and $v$, the consecutive vertices corresponding to $x_{1}$ alternately map to $t_{1}$ and $t_{2}$, etc.), and every (finite) tree homomorphic to $G$ is homomorphic to $T^{\prime}$.

To conclude, we restate some of the open problems suggested by this research.

1. Are there oriented trees $T$ which do not have bounded treewidth duality and for which the $T$-colouring problem is polynomial ? (We do not know any digraph with this property.)

2. Are there oriented trees $T$ such that the $T$-colouring problem is neither polynomial nor $N P$-complete ? (We again do not know this even for arbitrary digraphs. The answer is known to be negative for undirected graphs [17] and oriented cycles [10]; cf. also [11]. On the other hand, the class NP must contain such problems, unless $P=N P[26]$.)

3. Are there oriented trees $T$ which have bounded treewidth duality but do not have treewidth-2 duality? (Again, we do not know any digraph with this property.)

4. Prove that $T$ does not have bounded treewidth duality for at least some oriented tree $T$. (There are known digraphs with this property; in particular, it is shown in [32] that some oriented cycles do not have bounded treewidth duality. Our Theorem 4.5 shows oriented trees which do not have tree duality.)

Recent developments. In [11] the authors use another machinery for solving homomorphism problems in polynomial time, by using "datalog programs". Problems which can be solved this way are termed "of bounded width". We were informed by T. Feder that $H$ has bounded treewidth duality if and only if the $H$-colouring problem is of bounded width. Thus the class of $H$-colouring problems solvable in polynomial time is the same for the two techniques. Another recent approach that 


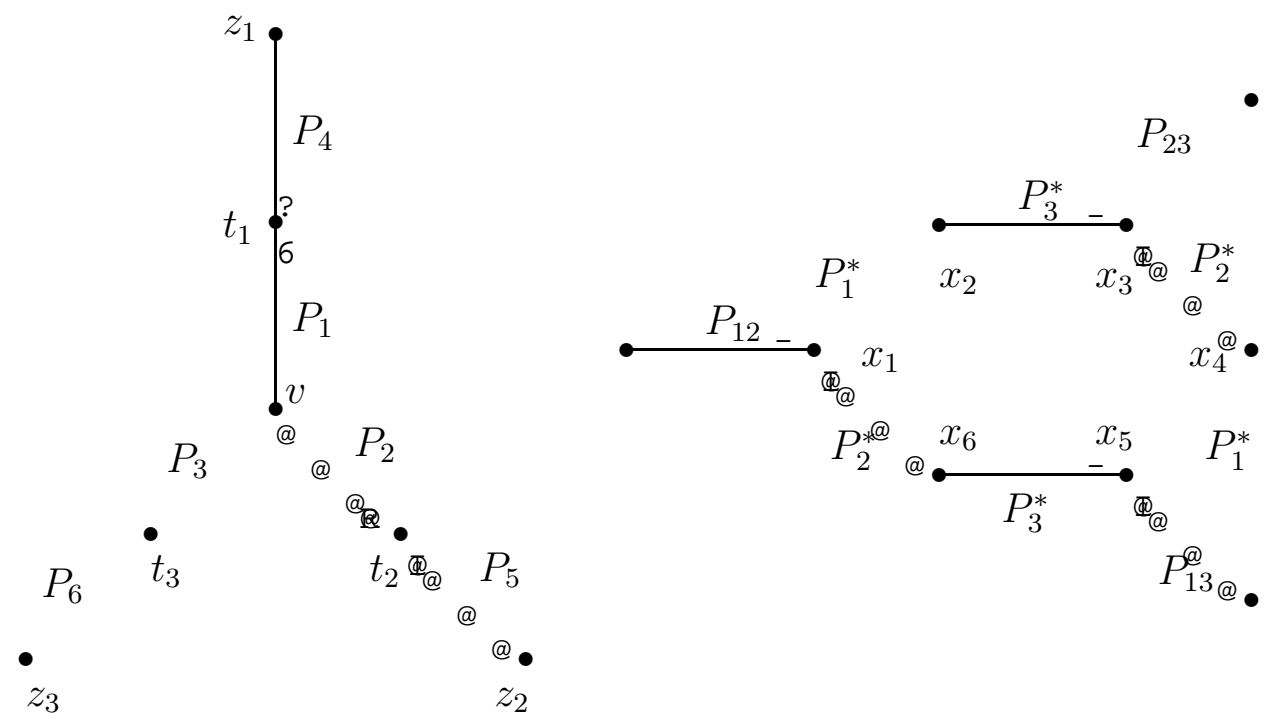

FiguRE 1.

A special triad $T$

Figure 2. A digraph $G$ for Theorem 4.5

can be used to solve $H$-colouring problems in polynomial time uses semidefinite programming [12]. It was recently shown in [2] that if $H$ has tree duality then the $H$-colouring problem can also be solved by this method (and a natural generalization of the method solves the $H$-colouring problems for digraphs $H$ with bounded treewidth duality).

\section{REFERENCES}

1. S. Arnborg, J. Lagergren, and D. Seese, Easy problems for tree-decomposable graphs, J. Algorithms 12 (1991), 308 - 340. MR 92d:05150

2. R. Bačik and S. Mahajan, Semidefinite programming and its applications to NP problems, manuscript, 1995.

3. J. Bang-Jansen and P. Hell, The effect of two cycles on the complexity of colourings by directed graphs, Discrete Applied Math. 26 (1990), 1-23. MR 91c:05072

4. J. Bang-Jansen, P. Hell and G. MacGillivray, The complexity of colourings by semicomplete digraphs, SIAM J. Discrete Math. 1 (1988), 281-289. MR 89e:05095

5. J. Bang-Jensen, P. Hell and G. MacGillivray, On the complexity of colouring by superdigraphs of bipartite graphs, Discrete Math. 109 (1992), 27 - 44. MR 93m:68051

6. J. Bang-Jensen, P. Hell and G. MacGillivray, Hereditarily hard colouring problems, Discrete Math. 138 (1995), 75-92.

7. J.A. Bondy and U.S.R. Murty, Graph theory with applications, American Elsevier, New York, 1976. MR 54:117

8. B. Courcelle, The monadic second-order logic of graphs, I: Recognizable sets of finite graphs, Information and Computation 85 (1990), 12 - 75. MR 91g:05107

9. R. Dechter, From local to global consistency, Artificial Intelligence 55 (1992), 87 - 107. MR 93d:68017

10. T. Feder, Classification of homomorphisms to oriented cycles (draft), manuscript, 1994.

11. T. Feder and M. Y. Vardi, Monotone monadic SNP and constraint satisfaction, 25th Annual ACM Syposium on Theory of Computing, 1993, 612-622. 
12. U. Feige and L. Lovász, Two-prover one-round proof systems: Their power and their problems, 24th Annual ACM Syposium on Theory of Computing, 1992, 733-744.

13. M. C. Golumbic, Algorithmic graph theory and perfect graphs, Academic Press, New York, 1980. MR 81e:68081

14. W. Gutjahr, Graph colourings, Ph. D. Thesis, Free University Berlin, 1991.

15. W. Gutjahr, E. Welzl and G. Woeginger, Polynomial graph colourings, Discrete Appl. Math. 35 (1992), 29-46.

16. R. P. Häggkvist and P. Hell, Universality of A-mote graphs, European J. of Combinatorics 14 (1993), 23-27. MR 94e:05251

17. P. Hell and J. Nešetřil, On the complexity of H-colouring, J. Combin. Th. (B) 48 (1990), 92-110. MR 91m:68082

18. P. Hell and J. Nešetřil, Homomorphisms of graphs and their orientations, Monatshefte für Math. 85 (1978), 39-48. MR 80a:05110

19. P. Hell, J. Nešetřil and X. Zhu, Duality and polynomial testing of tree homomorphisms, Technical Report, KAM series 93-243, Department of Applied Mathematics, Charles University, Prague, 1993.

20. P. Hell, J. Nešetřil and X. Zhu, Complexity of tree homomorphisms, Discrete Appl. Math., to appear.

21. P. Hell, J. Nešetřil and X. Zhu, Duality of Graph Homomorphisms, Combinatorics, Paul Erdös is Eighty, Vol. 2, Bolyai Society Mathematical Studies, Budapest (Hungary), 1995.

22. P. Hell and X. Zhu, The existence of homomorphisms to oriented cycles, SIAM J. on Disc. Math. 8 (1995), 208-222

23. P. Hell and X. Zhu, Homomorphisms to oriented paths, Discrete Math. 132 (1994), 107-114. MR 95:02

24. P. Hell, H. Zhou and X. Zhu, Homomorphisms to oriented cycles, Combinatorica 13 (1993), 421-433. MR 95d:05060

25. P. Komárek, Some new good characterizations of directed graphs, Časopis Pěst. Mat. 51 (1984), 348-354. MR 87a:05074

26. R. E. Ladner, On the structure of polynomial time reducibility, J. Assoc. Comput. Mach. 22 (1975), 155-171. MR 57:4623

27. G. MacGillivray, On the complexity of colourings by vertex-transitive and arc-transitive digraphs, SIAM J. Discrete Math. 4 (1991), 397-408. MR 92d:05156

28. H.A. Maurer, J.H. Sudborough and E. Welzl, On the complexity of the general colouring problem, Inform. and Control 51 (1981), 123-145. MR 84i:68073

29. U. Montanari, Networks of constraints: Fundamental properties and applications to picture processing, Inf. Sci. 7 (1974), 95 - 132. MR 54:1739

30. J. Nešetřil, Theory of graphs , SNTL (Praha), 1979

31. J. Nešetřil and A. Pultr, On classes of relations and graphs determined by subobjects and factorobjects, Discrete Math. 22 (1978), 287-300. MR 80k:05086

32. J. Nešetřil and X. Zhu, On Bounded Treewidth Duality of Graphs, manuscript, 1994.

33. N. Robertson and P.D. Seymour, Graph minors, II: Algorithmic aspects of treewidth, J. Algorithms 7 (1986), 309 - 322. MR 88c:05053

34. E. Welzl, Symmetric graphs and interpretations, J. Combin. Th. (B) 37 (1984), 235-244.

35. X. Zhu, A polynomial algorithm for homomorphisms to oriented cycles, J. of Algorithms, to appear. $1 \mathrm{~S} 6$

School of Computing Science, Simon Fraser University, Burnaby, BC, Canada V5A

E-mail address: pavol@cs.sfu.ca

Department of Applied Mathematics, Charles University, Prague, The Czech RepubLIC

E-mail address: nesetril@kam.ms.mff.cuni.cz

Sonderforschungsbereich 343, Universität Bielefeld, 33501 Bielefeld, Germany

E-mail address: xu@mathematik.uni-bielefeld.de 